\title{
295.
}

\section{ON THE CONSTRUCTION OF THE NINTH POINT OF INTER- SECTION OF THE CUBICS WHICH PASS THROUGH EIGHT GIVEN POINTS.}

[From the Quarterly Journal of Pure and Applied Mathematics. vol. v. (1862), pp. 222-233.]

I REPRODUCE with additional developments the solution which has been given of this interesting problem.

The equation of a given cubic may be written

$$
q U-p V=0
$$

where $U=0, V=0$ are any two conics meeting the cubic in the same four points; $p=0$ is the line joining the remaining two points of intersection of the cubic with the conic $U=0$; and $q=0$ is the line joining the remaining two points of intersection of the cubic with the conic $V=0$; the relation between the arbitrary constant factors implicitly contained in the functions $q U$ and $p V$ is assumed to be properly determined.

The form is employed by Plücker, Theorie der algebraischen Curven, p. 56 (1839), and in connexion therewith he gives some geometrical considerations which, he remarks, contain implicitly the solution of the above-mentioned problem.

The form is also the analytical basis of the investigations of $M$. Chasles, "Construction de la courbe du troisième ordre par neuf points," Comptes Rendus, t. xxxvi. (1853), pp. 942-952. In fact calling to mind the theorem that in the pencil of conics $U-\alpha V=0$ (where $\alpha$ is an arbitrary multiplier) the anharmonic ratio of the multipliers $\alpha$ is equal to the anharmonic ratio of the tangents at any one of the points of intersection, or (what is the same thing) to the anharmonic ratio of 
the polars of any point whatever in regard to the conics, and recollecting that the anharmonic ratio in question is said to be the anharmonic ratio of the conics themselves; then since the equation $q U-p V=0$ is satisfied by the system

$$
U-\alpha V=0, p-\alpha q=0,
$$

(where $\alpha$ is arbitrary) we have at once the theorem, p. 949 , viz. that if there be a pencil of lines through a point, and corresponding anharmonically thereto, a pencil of conics through the same four points; the locus of the intersections of a line by the corresponding conic is a cubic through the five points: and, conversely, that a given cubic may be so generated, the point of the pencil of lines, and the four points of the pencil of conics being any five points whatever of the cubic.

This gives at once the construction (M. Chasles' first construction) for the cubic through nine given points. In fact if the points are called 1, 2, 3, 4, 5, 6, 7, 8, 9; then grouping the points in any manner, it is only necessary to find a point $x$ such that

$$
x(1,2,3,4,5)=6789(1,2,3,4,5),
$$

that is, such that the pencil of lines $x 1, x 2, x 3, x 4, x 5$ shall correspond anharmonically to the pencil of conics $67891,67892,67893,67894,67895$. The foregoing notation is that employed in M. de Jonquières' "Essai sur la génération des Courbes géométriques, \&c.," Mém. Sav. Etrang., t. XVI. (1858), which I take the opportunity of referring to. In fact, if $x$ satisfies the foregoing condition, then taking through the point $x$ any other line, and corresponding : anharmonically thereto a conic through the points $6,7,8,9$, the locus of the intersections of the line and conic will be a cubic through the nine points. But the condition in question gives

$$
\begin{aligned}
& x(1,2,3,4)=6789(1,2,3,4), \\
& x(1,2,3,5)=6789(1,2,3,5),
\end{aligned}
$$

which (by the anharmonic property of the points of a conic) show, the first that $x$ is in a certain conic passing through $1,2,3,4$, and the second that $x$ is in a certain conic passing through $1,2,3,5$; the two conics intersect in the points $1,2,3$, and in a fourth point which is the required point $x$. Or we may say that $x$ is given by the condition that the pencils $x(1,2,3,4)$ and $x(1,2,3,5)$ shall have given anharmonic ratios. It will presently be seen how $x$ can be determined by the ruler alone.

Suppose now that the points $1,2,3,4,5,6,7,8,9$ are the points of intersection of two cubics; the construction should become indeterminate; this is only the case when the two conics which by their intersection should determine $x$ become one and the same conic. This implies that the conic $x(1,2,3,4)$ passes through 5 , or that $x, 1,2,3,4,5$ are points of the same conic. And then since by the anharmonic property of the points of a conic $x(1,2,3,4)=5(1,2,3,4)$, we have

$$
5(1,2,3,4)=6789(1,2,3,4) \text {. }
$$


The grouping of the nine points is altogether arbitrary, hence there are in all $(9 \times 70=) 630$ such equations, which are really equivalent to only two equations, and which when eight of the points are given, determine the ninth point. Supposing. that the given points are $1,2,3,4,5,6,7,8$, the equations for the determination of the remaining point 9 may be taken to be

$$
\begin{aligned}
& 9(5,6,7,8)=1234(5,6,7,8), \\
& 9(4,6,7,8)=1235(4,6,7,8),
\end{aligned}
$$

which (it is to be remarked) determine 9 in a similar way to that in which $x$ is given in the construction of the cubic through nine points; viz. 9 is the fourth intersection of two conics which pass through the points 5, 6, 7, 8 and the points $4,6,7,8$ respectively. Or we may say that 9 is given by the conditions that the pencils $9(5,6,7,8)$ and $9(4,6,7,8)$ shall have given anharmonic ratios.

The foregoing equations

$$
\begin{aligned}
& 9(5,6,7,8)=1234(5,6,7,8), \\
& 9(4,6,7,8)=1235(4,6,7,8),
\end{aligned}
$$

are equivalent to and constitute the geometrical interpretation of the equations obtained (previous to M. Chasles' Memoir) by Weddle in the paper "On the construction of the ninth point of intersection of two curves of the third degree when the other eight points are given," Cambridge and Dublin Mathematical Journal, t. vI., pp. 83-86 (1851). In fact, reproducing his analysis with only a slight change of notation, let 012 denote the determinant

$$
\left|\begin{array}{lll}
x, & y, & z \\
x_{1}, & y_{1}, & z_{1} \\
x_{2}, & y_{2}, & z_{2}
\end{array}\right|
$$

so that $012=0$ is the equation of the line through the points 1 and 2 ; and in like manner let 012345 denote the determinant

$$
\left|\begin{array}{l}
x^{2}, y^{2}, z^{2}, y z, z x, x y \\
x_{1}^{2}, \& c . \\
\vdots
\end{array}\right|
$$

so that $012345=0$ is the equation of the conic through the points $1,2,3,4,5$. Of course 123, 123456, \&c. will denote given functions of the coordinates of the points $1,2,3$, the points $1,2,3,4,5,6$, \&c. This being so

$$
012345.078=\lambda .012347 .058
$$

is the equation of a particular cubic passing through the points $1,2,3,4,5,7,8$, and which if we properly determine $\lambda$, viz. if we write

$$
\lambda=\frac{612345.678}{612347.658}
$$

will also pass through the point 6 .

c. IV. 
And similarly

$$
012345.076=\mu .012347 .056
$$

is the equation of a particular cubic curve passing through the points $1,2,3,4,5,6,7$ and which if we properly determine $\mu$, viz. if we write

$$
\mu=\frac{812345.876}{812347.856}
$$

will also pass through the point 8. Hence the two curves, each of them passing through the points $1,2,3,4,5,6,7,8$ will intersect in the remaining point 9 ; and writing 9 for 0 , and combining the two equations, we have

or, what is the same thing,

$$
\frac{978.956}{958.976}=\frac{\lambda}{\mu}=\frac{612345}{612347} \frac{812347}{812345},
$$

$$
\frac{956.978}{958.967}=\frac{123456.123478}{123458.123467},
$$

which is Weddle's equation, and is equivalent to the above-mentioned equation

$$
9(5,6,7,8)=1234(5,6,7,8) \text {. }
$$

To prove this I remark that we have identically

$$
012.034 .514 .523-014.023 .512 .534=012345 \text {. }
$$

In fact the left-hand side equated to zero is the equation of the conic through $1,2,3,4,5$, and such left-hand side must therefore, save to a mere numerical factor, be equal to 012345 . And to determine this factor it is to be observed that 012345 contains the term

$$
+x_{0}^{2} \cdot y_{1}^{2} \cdot z_{2}^{2} \cdot y_{3} z_{3} \cdot z_{4} x_{4} \cdot x_{5} y_{5},
$$

but that there is no such term in 012.034.514.523, and that there is in - 014.023.512.534 the equivalent term

$$
-x_{0} y_{1} z_{4} \cdot-x_{0} y_{3} z_{2} \cdot x_{5} y_{1} z_{2} \cdot y_{5} z_{3} x_{4},
$$

so that the numerical factor is rightly determined.

The foregoing identity written under the form

$$
\frac{012.034}{014.023}-\frac{512.534}{514.523}=\frac{012345}{014.023 .514 .523}
$$

shows that, when $012345=0$, i. e. if 0 be a point of the conic through $1,2,3,4,5$, then we have

$$
0(1,2,3,4)=5(1,2,3,4) \text {, }
$$

which is in fact the anharmonic property of the points of a conic. And observing that $012345=051234$, and substituting 5,6 , for 0,5 respectively, the identity becomes

$$
\frac{512.534}{514.523}-\frac{612.634}{614.623}=\frac{561234}{514.523 .614 .623} \text {. }
$$


The equation $012345=0$ may be written

$$
012.034-\frac{512.534}{514.523} 014.023=0
$$

and hence the anharmonic ratio of the conics

$$
012345=0,012346=0,012347=0,012348=0
$$

is equal to that of the quantities

$$
\frac{512.534}{514.523}, \frac{612.634}{614.623}, \frac{712.734}{714.723}, \frac{812.834}{814.823},
$$

or calling these quantities for a moment $\alpha, \beta, \gamma, \delta$, it is

where

$$
=\frac{(\alpha-\beta)(\gamma-\delta)}{(\alpha-\delta)(\beta-\gamma)},
$$

$$
\alpha-\beta=\frac{512.534}{514.523}-\frac{612.634}{614.623}=\frac{561234}{514.523 .614 .623} ;
$$

and forming in this manner the expressions of each of the four factors $\alpha-\beta, \gamma-\delta$, $\alpha-\delta, \beta-\gamma$, we have

$$
\frac{(\alpha-\beta)(\gamma-\delta)}{(\alpha-\delta)(\beta-\gamma)}=\frac{561234.781234}{581234.671234},
$$

so that in the equation

the right-hand side is

$$
\frac{956.978}{958.967}=\frac{561234.781234}{581234.671234},
$$

$$
=1234(5,6,7,8) \text {, }
$$

and since by what precedes the left-hand side is $=9(5,6,7,8)$, the equation is

$$
9(5,6,7,8)=1234(5,6,7,8),
$$

which is the transformation in question.

Now resuming the two equations

$$
\begin{aligned}
& 9(5,6,7,8)=1234(5,6,7,8), \\
& 9(4,6,7,8)=1235(4,6,7,8),
\end{aligned}
$$

the right-hand sides are given anharmonic ratios, and as we have seen the question is to find 9 so that the anharmonic ratios $9(5,6,7,8), 9(4,6,7,8)$ shall have given values. But for the geometrical solution by the ruler alone, we have the preliminary question, from the given eight points, without the assistance of the before-mentioned conics, to construct the given anharmonic ratios $1234(5,6,7,8)$ and $1235(4,6,7,8)$. The solution of both questions is given in Dr Hart's paper, "Construction by the ruler alone to determine the ninth point of intersection of two curves of the third degree," Cambridge and Dublin Mathematical Journal, t. vI., pp. 181, 182 (1851). 
The Preliminary Question. The anharmonic ratio $1234(5,6,7,8)$ is equal to that of the polars of an arbitrary point $X$ in regard to the conics 1234.5, 12346, 12347, 12348 respectively (these polars all pass through one and the same point). Now to construct the polars of $X$ in regard to these conics, and first in regard to the conic 12345: the fourth harmonics of $X$ in regard to the lines 12, 34, in regard to the lines 13, 42, and in regard to the lines 14, 23, meet in a point; and considering the several combinations $1234,1235,1245,1345,2345$ we have thus five points; these lie on a line which is the required polar of $X$ in regard to the conic 12345. The polars in regard to the other conics are obtained in the same manner; and it is clear that the first above-mentioned point (viz. that deduced from the points $1,2,3,4$ ) is in fact the point of intersection of the four polars, or point of the pencil formed by the polars.

The Principal Question then is, given the points 4, 5, 6, 7, 8 to find the point 9, such that

$$
\begin{aligned}
& 9(5,6,7,8)=1234(5,6,7,8) \\
& 9(4,6,7,8)=1235(4,6,7,8)
\end{aligned}
$$

where the right-hand sides represent given anharmonic ratios.

For this, let 65,74 (see the figure) meet in $M$, and on 74 find a point $Q$ such

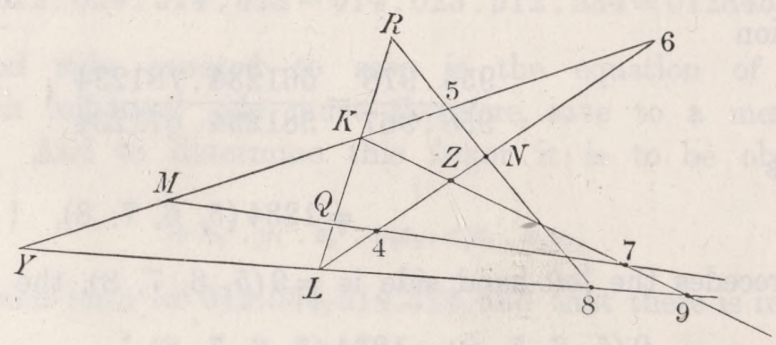

that the anharmonic ratio of the points $(4, M, 7, Q)$ may be equal to the given ratio $1235(4,6,7,8)$, say

$$
(4, M, 7, Q)=1235(4,6,7,8)
$$

and let 64,85 meet in $N$ and on 85 find a point $R$, such that the anharmonic ratio of the points $5, N, R, 8$ is equal to the given anharmonic ratio $1234(5,6,7,8)$, say

$$
(5, N, R, 8)=1234(5,6,7,8) \text {. }
$$

Join $Q R$ meeting 65 in $K$ and 64 in $L$; then $7 K$ and $8 L$ will meet in the required point 9 .

In fact taking $Y$ as the intersection of the lines $65 K M$ and $8 L$, and $Z$ as the intersection of the lines $64 N L$ and $7 K$; then, as is clear from the figure, first, the 
anharmonic ratio $9(4,6,7,8)$ is equal to that of the points $(4,6, Z, L)$ on the line $46 Z L$, that is

which is

$$
9(4,6,7,8)=(4,6, Z, L),
$$

$$
=(4, M, 7, Q) \text {, }
$$

since the lines $6 M, Z 7, L Q$ meet in the point $K$; but by the construction $(4, M, 7, Q)=1235(4,6,7,8)$, that is, we have

$$
9(4,6,7,8)=1235(4,6,7,8) \text {; }
$$

and, secondly, the anharmonic ratio $9(5,6,7,8)$ is equal to that of the points $(5,6, K, Y)$ on the line $56 K Y$, that is

$$
9(5,6,7,8)=(5,6, K, Y),
$$

which is

$$
=(5, N, R, 8) \text {, }
$$

since the lines $6 N, K R, Y 8$ meet in the same point $L$; but by the construction $(5, N, R, 8)=1234(5,6,7,8)$, that is, we have

$$
9(5,6,7,8)=1234(5,6,7,8),
$$

so that the point 9 satisfies the required conditions.

It has been already remarked that the point $x$ in M. Chasles' theorem for the construction of the cubic through nine points is determined by precisely similar conditions to those which determine the ninth intersection of the two cubics; that is, the foregoing construction by the ruler alone is applicable to the determination of the point $x$; and when this is once obtained, the remainder of the construction, giving the points of the cubic through the nine given points, can obviously be performed by the ruler alone. The construction for the cubic through nine points gives implicitly the relation between ten points of the cubic and such relation is accordingly expressed by the equation

$$
x(1,2,3,4,5,10)=6789(1,2,3,4,5,10),
$$

which is one out of 210 similar forms. But it is possible that some more convenient form of the relation between the ten points may yet be found.

I proceed to further develope the analytical theory. Writing for convenience $\omega$ in the place of 10 , we have

$$
x(1,2,3,4,5,6)=789 \omega(1,2,3,4,5,6),
$$

or, what is the same thing,

$$
\begin{aligned}
& x(1,2,3,4)=789 \omega(1,2,3,4), \\
& x(1,2,3,5)=789 \omega(1,2,3,5), \\
& x(1,2,3,6)=789 \omega(1,2,3,6),
\end{aligned}
$$


which belong to three conics each of them passing through 1, 2, 3, and which must have a remaining fourth point of intersection.

The equation of the first conic is

where

$$
012.034=\frac{\lambda}{\mu} 014.023
$$

$$
\begin{aligned}
& \lambda=789 \omega 12.789 \omega 34, \\
& \mu=789 \omega 14.789 \omega 23,
\end{aligned}
$$

and thence, in virtue of an identical equation already referred to,

$$
-\lambda-\mu=789 \omega 13.789 \omega 24 \text {. }
$$

But we have identically

$$
123.0 p q=1 p q .023+2 p q .031+3 p q .012,
$$

and thence in particular

$$
\begin{aligned}
& 123.034=134.023+234.031, \\
& 123.014=214.031+314.012,
\end{aligned}
$$

and the equation of the conic may therefore be written

that is

$$
012(134.023+234.031) \mu-023(214.031+314.012) \lambda=0,
$$

$$
031.012 .234 . \mu+012.023 .314(-\lambda-\mu)+023.031 .124 . \lambda=0 \text {, }
$$

or, substituting for $\mu,-\lambda-\mu, \lambda$, their values, this is

$$
\begin{aligned}
& 031.012 .234 .789 \omega 14.789 \omega 23 \\
+ & 012.023 .314 .789 \omega 13.789 \omega 42 \\
+ & 023.031 .124 .789 \omega 12.789 \omega 34=0
\end{aligned}
$$

or, what is the same thing,

$$
\frac{234.789 \omega 14.789 \omega 23}{023}+\frac{314.789 \omega 13.789 \omega 24}{031}+\frac{124.789 \omega 12.789 \omega 34}{012}=0,
$$

or, making a slight change of form, the equation of the conic is

$$
\frac{423.789 \omega 41.789 \omega 23}{023}+\frac{431.789 \omega 42.789 \omega 31}{031}+\frac{412.789 \omega 43.789 \omega 12}{012}=0 \text {. }
$$

The equations of the other two conics are deduced by writing successively 5 and 6 in the place of 4 ; and the condition in order that the conics may have a remaining fourth point of intersection is

$$
\begin{array}{lll}
423.789 \omega 41, & 431.789 \omega 42, & 412.789 \omega 43 \\
523.789 \omega 51, & 531.789 \omega 52, & 512.789 \omega 53 \\
623.789 \omega 61, & 631.789 \omega 62, & 612.789 \omega 63
\end{array} \mid=0 .
$$


This equation, say $\square=0$, expresses the relation between the coordinates of the ten points $1,2,3,4,5,6,7,8,9, \omega$, of the cubic. Hence if $123456789 \omega$ denote the determinant

$$
\mid \begin{aligned}
& x_{1}^{3}, \quad y_{1}^{3}, z_{1}^{3}, \quad y_{1}^{2} z_{1}, \quad z_{1}^{2} x_{1}, \quad x_{1}^{2} y_{1}, y_{1} z_{1}^{2}, z_{1} x_{1}^{2}, \quad x_{1} y_{1}^{2}, x_{1} y_{1} z_{1} \\
& x_{2}^{3}, \& c .
\end{aligned}
$$

$123456789 \omega$ must be a factor of $\square$, and a little consideration shows that the other factor which is of the order one as regards the coordinates of each of the points $1,2,3$, and of the order three as regards the coordinates of each of the points $7,8,9, \omega$, must be of the form $123.789 .78 \omega .79 \omega .89 \omega$. We must therefore have

$$
\square=\epsilon .123 .789 .78 \omega .79 \omega .89 \omega .123456789 \omega,
$$

where the merely numerical factor $\epsilon$ is, I believe, equal to +1 or else to -1 .

In order to verify the factor $123.789 .78 \omega .79 \omega .89 \omega$, observing that the points $7,8,9$, $\omega$ enter symmetrically, it will be sufficient to show that 123,789 are each of them factors of $\square$, or, what is the same thing, that if $123=0$, or if $789=0$, then in either case $\square=0$.

First, if $123=0$, we may write

equations which give

$$
\begin{aligned}
& x_{3}=\lambda x_{1}+\mu x_{2}, \\
& y_{3}=\lambda y_{1}+\mu y_{2}, \\
& z_{3}=\lambda z_{1}+\mu z_{2}
\end{aligned}
$$

$$
423=\lambda .421,431=\lambda .421 \text {, \&c., }
$$

and the equation $\square=0$, thus becomes

$$
\begin{array}{lll}
789 \omega 41, & 789 \omega 42, & 789 \omega 43 \\
789 \omega 51, & 789 \omega 52, & 789 \omega 53 \\
789 \omega 61, & 789 \omega 62, & 789 \omega 63
\end{array} \mid=0,
$$

or, what is the same thing,

$$
\begin{array}{lll}
789 \omega 14, & 789 \omega 24, & 789 \omega 34 \\
789 \omega 15, & 789 \omega 25, & 789 \omega 35 \\
789 \omega 16, & 789 \omega 27, & 789 \omega 36
\end{array} \mid=0 .
$$

Now if the terms in the same column are multiplied by $789 \omega 56,789 \omega 64,789 \omega 45$ respectively and added, then for the first column the sum is

$$
789 \omega 14.789 \omega 56+789 \omega 15.789 \omega 64+789 \omega 16.789 \omega 4 \check{\check{\rho}}
$$

which is $=0$, and the sums for the second and third columns are each $=0$ in the same manner: wherefore the determinant vanishes as it should do. 
Next, if $789=0$, we have identically

$$
789 \omega 41=789.741 . \omega 81 . \omega 94+781.794 \cdot \omega 89 \cdot \omega 41,
$$

which when $789=0$ gives

$$
789 \omega 41=781.794 \cdot \omega 89 \cdot \omega 41
$$

and in like manner,

$$
\begin{aligned}
& 789 \omega 42=782.794 \cdot \omega 89 \cdot \omega 42, \\
& 789 \omega 43=783.794 \cdot \omega 89 \cdot \omega 43,
\end{aligned}
$$

in which three equations 4 may be changed into 5 and 6 successively. The equation $\square=0$ thus becomes

$$
\begin{array}{lll}
423 . \omega 41, & 431 . \omega 42, & 412 . \omega 43 \\
523 . \omega 51, & 531 . \omega 52, & 512 . \omega 53 \\
623 . \omega 61, & 631 . \omega 62, & 612 . \omega 63
\end{array} \mid=0,
$$

or, what is the same thing,

$$
\begin{array}{lll}
423.41 \omega, & 421.4 \omega 3, & 42 \omega .431 \\
523.51 \omega, & 521.5 \omega 3, & 52 \omega .531 \\
623.61 \omega, & 621.6 \omega 3, & 69 \omega .631
\end{array} \mid=0,
$$

and since the sum of the terms in each line of the determinant is $=0$, the determinant is as it should $b e=0$.

The foregoing equation

$$
\begin{aligned}
& 412.789 \omega 43, \quad 423.789 \omega 41,-431.789 \omega 42 \\
& 512.789 \omega 53, \quad 523.789 \omega 51, \quad 531.789 \omega 52 \\
& 612.789 \omega 63, \quad 623.789 \omega 61, \quad 631.789 \omega 62 \\
& =\epsilon .123 .789 .78 \omega .79 \omega .89 \omega .123456789 \omega \text {, }
\end{aligned}
$$

(since 412, $789 \omega 43$, \&c. are interpretable functions of the coordinates) affords a geometrical interpretation of the equation

$$
123456789 \omega=0
$$

between the coordinates of the ten points of the cubic; but it would be more satisfactory if a similar identical equation could be found, having on the right-hand side the function $123456789 \omega$ without the irrelevant factor

$$
123.789 .78 \omega .79 \omega .89 \omega .
$$

2, Stone Buildings, W.C., 6th March, 1862. 